\title{
Vínculo entre percepción territorial y movilidad espacial en trayectorias de la clase media profesional: región de Los Lagos, Chile
}

\author{
The link between territorial perception and spatial \\ mobility in trajectories of the professional \\ middle-class: Los Lagos region, Chile
}

Felipe Andrés Saravia ${ }^{[l]}$

\section{Resumen}

Se buscó identificar los contenidos de las percepciones subjetivas que la clase media profesional originaria de la región de Los Lagos tiene sobre sus territorios de origen, y cómo se vinculan con estrategias de movilidad espacial en tres momentos de sus trayectorias de vida: formación secundaria, acceso a la educación universitaria y etapa de inserción laboral. Se analizaron cualitativamente 43 entrevistas con el software Atlas.ti, ahondando en la relación entre ciudades intermedias e hinterland, y región de origen con regiones con presencia de ciudades metropolitanas. Se observa que las percepciones se asocian a estrategias concretas de movilidad espacial, y que los niveles territoriales de las tensiones, fuerzas centrifugas y centrípetas, varían según el momento del ciclo vital de los sujetos.

Palabras clave: percepción subjetiva del territorio; movilidad espacial; clase media profesional; trayectorias de vida; ciudades intermedias.

\begin{abstract}
We aimed to identify the contents of subjective perceptions that the professional middle-class of the Los Lagos region has of their territories of origin, and how they are linked to spatial mobility strategies in three moments of their life trajectories: secondary education, access to university education and placement in the job market. Overall, 43 interviews were analyzed qualitatively using the Atlas.ti software. We investigated the relationship between intermediate and hinterland cities, and between region of origin and regions with the presence of metropolitan cities. We found that the perceptions are associated with specific spatial mobility strategies, and that the territorial levels of the tensions, centrifugal and centripetal forces, vary according to the moment of the subjects' life cycle.
\end{abstract}

Keywords: subjective perception of the territory; spatial mobility; professional middle-class; life trajectories; intermediate cities. 


\section{Introducción}

El presente artículo aborda como objeto de análisis el vínculo entre percepciones subjetivas de territorios y estrategias de movilidad espacial de la clase media profesional. Se plantea como preguntas centrales cuáles son los contenidos de las percepciones subjetivas que la clase media profesional originaria de la región de Los Lagos tiene respecto de sus territorios de origen, y cómo estas se vinculan con las estrategias de movilidad espacial en las trayectorias de vida de los sujetos, específicamente en tres momentos de estas: 1) durante la enseñanza media, 2) durante el acceso a la educación universitaria y 3 ) posterior al egreso de esta, en la etapa de inserción laboral.

Se analiza el caso específico de quienes rindieron la Prueba de Selección Universitaria (en adelante PSU) entre los años 2004 y 2006 en la región de Los Lagos, en Chile, que fueron seleccionados en universidades chilenas y que en la actualidad tienen entre 4 y 8 años de experiencia laboral. El foco del análisis está puesto en la asociación entre las estrategias de movilidad espacial inter-regional por motivos de estudios universitarios de pregrado que los sujetos despliegan, y la percepción subjetiva que respecto de sus territorios de origen tienen. Fueron entrevistados sujetos que actualmente residen en sus territorios de origen y otros que emigraron.

La preocupación por esta temática es considerada relevante porque a pesar de que a nivel internacional existen desde hace décadas tradiciones de investigación sobre imaginarios urbanos (Hiernaux, 2007) o geografías de las percepciones (Vara, 2008), en Chile no ha sido suficientemente abordada la relación entre subjetividades territoriales y movilidad espacial (Rodrigo y Atienza, 2014; Hiernaux, 2007), por lo que se considera que este trabajo viene a contribuir a la comprensión de fenómenos cuya aproximación desde las ciencias sociales y de la política pública, tiende a ser hecha desde el sentido común. Como plantea Wacquant (2017, p. 291)

[...] vastos sectores de la investigación urbana aceptan la terminología, las preguntas y las preocupaciones planteadas por administradores locales, tomadores de decisión, periodistas 0 la moda académica, cuando deberían en cambio detectar y neutralizar el inconsciente histórico y los sesgos sociales incorporados en ellos mismos.

El caso analizado es el de la región de Los Lagos, ubicada a poco más de 900 kilómetros al sur de la capital nacional. Tenía una población estimada el año 2004 de 762.172 habitantes, y de 853.663 para el 2017 (INE, 2017). En términos político-administrativos, se configura a partir de cuatro provincias (Osorno, Llanquihue, Chiloé y Palena), al interior de las cuales se distribuyen 30 comunas. En este trabajo se desarrolla el análisis de las percepciones agrupando comunas que pueden ser consideradas ciudades intermedias y el resto que corresponden a sus zonas de influencia. Los resultados pretenden aportar tanto a la comprensión de la región en análisis, como al estudio en general de las subjetividades en ciudades intermedias e hinterland.

A continuación, el artículo siguiente el siguiente esquema: en primer lugar, se desarrolla un marco referencial que primeramente se 
refiere a los aspectos estructurales de los territorios analizados, específicamente su configuración interna, haciendo especial énfasis en el rol de las ciudades intermedias y su vínculo con sus correspondientes zonas de influencia. Luego se fundamenta la relevancia de analizar las percepciones subjetivas de personas comunes y corrientes respecto del territorio, y por último se hace referencia a las estrategias de acción espacial analizadas, abordando tanto la movilidad espacial por estudios universitarios, como las estrategias de movilidad espacial de profesionales. A partir de esta revisión de literatura científica, se desarrollan los supuestos. Posteriormente se detalla la metodología utilizada, para en un cuarto acápite dar cuenta de los resultados, y finalmente algunas reflexiones a la luz de estos.

\section{Estructura: ciudades intermedias e hinterland}

Las ciudades intermedias constituyen un objeto de análisis relevante, no solo porque concentran más de la mitad de la población mundial (Unesco, 1999; Bellet y Llop, 2004), sino también porque cumplen un rol de intermediación en los sistemas de ciudades y en sus respectivas áreas de influencia o hinterland, que les convierte en nodos centrales en las dinámicas de los territorios no metropolitanos (Maturana, 2016) y a nivel nacional (Lufin y Atienza, 2015), y porque en Chile han tenido durante las últimas seis décadas, un dinamismo demográfico similar en el caso de aquellas con tamaño poblacional de 50.000 a 100.000 habitantes - o mayor - en el caso de aquellas de tamaño poblacional de 100.000 a 500.000 habitantes - que el de las ciudades metropolitanas (Rodriguez, Gonzalez y Ojeda, 2009).

Ahora bien, el término ciudad intermedia no es fácilmente definible (Unesco, 1999) y no existe consenso sobre su conceptualización. Más bien, se propone que su definición deberá variar dependiendo del contexto en el que estas se insertan (Bellet y Llop, 2004), ya que precisamente el término "media" 0 "intermedia" hace referencia a una definición por una doble contraposición. No son grandes metrópolis, pero tampoco son ciudades pequeñas (Taulelle, 2015). Como plantean Bellet y Llop (2004) "una ciudad media o intermedia en Europa se correspondería con una ciudad pequeña y/o muy pequeña en el contexto chino o indio, en que hay un importante número de ciudades que superan fácilmente el millón de habitantes". Sin embargo, una definición ampliamente aceptada es que son asentamientos humanos con una población superior a 50.000 habitantes, que se vinculan con estructuras mayores como las grandes ciudades y con un entorno integrado por núcleos urbanos más pequeños y zonas semirurales o rurales, al mismo tiempo que constituyen centros proveedores de servicios relativamente especializados, son nodos en la infraestructura de transporte y albergan una interacción social, política y cultural relativamente heterogénea (Jordán y Simioni, 1998; Valicelli y Pesci, 2002).

De acuerdo a esta lógica y teniendo en consideración la tipología elaborada por Maturana (2015) para el caso chileno que incluye diversos criterios adicionales que van más allá del tamaño poblacional, 
se consideraron tres ciudades intermedias: Osorno, Puerto Montt y Castro. En el caso de las dos últimas, se consideran agrupadas con comunas aledañas. Puerto Montt se asocia a Puerto Varas y Llanquihue, y Castro a Dalcahue y Chonchi. Entre estas comunas se produce lo que Coombes y Bond (2008) y Casado-Díaz y Coombes (2011) denominan zonas de mercado laboral local, determinadas mediante una metodología que a partir de la medición de flujos de conmutación identifica áreas que permiten satisfacer necesidades de trabajo, vivienda, infraestructura y servicios sin necesariamente desplazarse fuera de ese lugar, y que no siempre responden solo a una unidad territorial delimitada en términos político-administrativos. Metodologías similares han sido aplicadas en Chile por Berdegué y otros (2011), Maturana y Arenas (2012), Fuentes, Mac-Clure, Moya y Olivos (2017), Casado-Díaz, Bernabéu y Rowe (2017) y Rowe, Casado-Díaz y Martínez-Bernabéu

Figura 1 - Territorios intrarregionales de Los Lagos

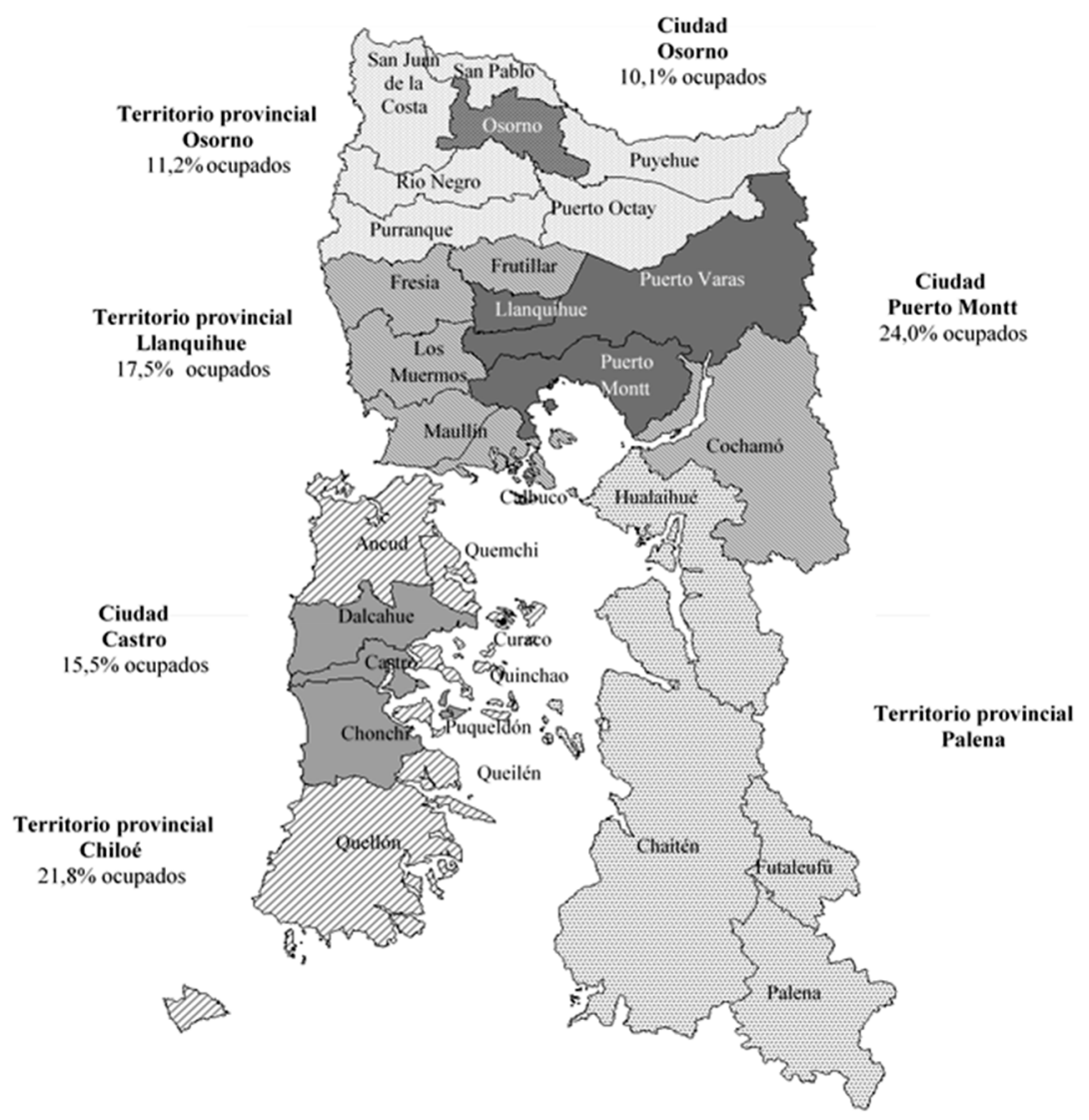

Fuente: elaboración propia, en base a Casen (2015). 
(2017). La revisión de los resultados del trabajo de Mac-Clure y Calvo (2013), en el que categorizan todas las comunas del país en distintos tipos de territorios según niveles de conmutación inter-comunal, más los resultados del estudio de Lufin y Atienza (2015) en lo referido a Puerto Montt y Puerto Varas, permiten fundamentar esta decisión teórico-metodológica.

De esta manera, como se observa en la Figura 1, la región de Los Lagos se configura en la interacción entre tres ciudades intermedias y sus correspondientes zonas de influencia, más la zona de Palena que no cuenta con ciudad intermedia, se encuentra escasamente poblada y se ubica más alejada del resto de las ciudades de la región, razón por la que no se considera en el análisis.

\section{Percepciones subjetivas territoriales}

Este trabajo se centra en las percepciones subjetivas sobre territorios a nivel regional e intrarregional, niveles de delimitación espacial que en lo que concierne a las ciencias sociales, predominantemente han sido abordados desde la mirada experta, tal como plantea Bourdieu (2006, p. 2):

[...] la región es una apuesta de luchas entre sabios, obviamente geógrafos, que relacionados con el espacio, pretenden naturalmente el monopolio de la definición legítima, pero también historiadores, etnólogos, y sobretodo desde que existe una política de regionalización, y de los movimientos regionalistas, economistas y sociólogos.
Estas aproximaciones legitimadas desde el saber experto de diversas disciplinas, no siempre han puesto suficiente énfasis en cómo la construcción subjetiva del territorio es también realizada por los sujetos comunes y corrientes que allí habitan. Al respecto Bourdieu (2006) plantea que la importancia dada a los fenómenos físicos en los análisis espaciales, ha ido en desmedro de la consideración de otras dimensiones de análisis como la acción del Estado, movimientos de capitales, o decisiones de grupos, entre otras. Al contrario, Bourdieu propone "reconceptualizar lo urbano como dominio de acumulación, diferenciación y disputa de múltiples formas de capital" (Wacquant, 2017, p. 299). No obstante, en palabras de Soja (2008), el foco predominante ha estado puesto en el análisis de lo que denomina como primer espacio.

Soja (2008) se refiere a la dimensión espacial desde una trialéctica, compuesta por tres aspectos del espacio. El primer espacio hace referencia a la materialidad de este, es decir, se asocia a aquello que puede ser "percibido física y empíricamente como forma y proceso, como configuraciones y prácticas de la vida urbana plausibles de ser medidas y cartografiadas" (Soja, 2008, p. 39). El segundo espacio en cambio, se ocupa más bien de aspectos subjetivos como pensamientos, representaciones simbólicas, 0 imágenes mentales acerca del espacio, siendo denominado por el autor como imaginario urbano. $A$ estos tres aspectos suma un tercer espacio, en el que convergen los dos anteriores. Se trata de "un lugar simultáneamente real e imaginario, actual y virtual, lugar de experiencia y agencia estructuradas, individuales y colectivas" (Soja, 2008, p. 39). 
Es al segundo y tercer espacio descrito por Soja al que se aboca la investigación aquí presentada. Interesa en primer lugar comprender cuáles son los elementos que componen la percepción subjetiva de los territorios de origen de los sujetos, y en segundo lugar, cómo dichas percepciones se asocian a acciones respecto del territorio, específicamente, la movilidad espacial.

El estudio sobre la dimensión subjetiva del espacio tiene una historia que se remonta a al menos cinco décadas, pero se ha enfocado principalmente en el ámbito urbano (Vara, 2008), y salvo escasas excepciones (Morales, 2012), ha analizado grandes ciudades o metrópolis, por lo que se considera que este estudio al analizar la relación entre ciudades intermedias e hinterland, y entre una región apartada del centro nacional como la región de Los Lagos, con el resto del país, significa una contribución a esta línea de investigación.

La configuración territorial descrita en el apartado anterior, permite aproximarse al análisis de las representaciones subjetivas del territorio desde distintos niveles o sentidos. Soja (2008) propone que el espacio urbano opere en al menos tres: desde la ciudad hacia su interior inmediato (nivel intraurbano), desde cada ciudad hacia otras de tamaño similar (nivel interurbano), y desde ciudades hacia sus correspondientes zonas de influencia 0 hinterland. Para efectos de este trabajo nos referiremos al tercer nivel de análisis que contrapone a las tres ciudades intermedias identificadas con sus correspondientes zonas de influencia, al cual se adicionará un nivel más general que contrapone la región en su conjunto con el resto del país.
En estos dos niveles se analiza, a partir de las subjetividades de los entrevistados, las tensiones entre lo que Soja (2008) denomina como fuerzas de aglomeración y centralización - centrípetas -, y de expulsión y descentralización - centrífugas -, las que operan simultáneamente de forma diversa en distintas escalas y en distintos ámbitos. En particular, se analiza cómo estas dos fuerzas contradictorias entre sí, propician estrategias de movilidad espacial por parte de los sujetos entrevistados, en tres momentos: durante su enseñanza media, durante el acceso a la educación universitaria, y posterior al egreso de esta.

Las fuerzas centrípetas y centrífugas son conceptualizadas acá como fenómenos que se inscriben en los tres tipos de lugares descritos por Soja. Es decir, corresponden tanto a aspectos materiales, como a procesos subjetivos en relación con el espacio y los territorios, y a la interacción entre estas dos dimensiones a través de estrategias de movilidad espacial desplegadas por los sujetos. Estas operan en distintos niveles de tensión territorial. En este caso se analiza cómo operan en las tensiones que se produce entre ciudades intermedias e hinterland y entre la región de Los Lagos y las regiones con presencia de ciudades metropolitanas.

\section{Estrategias de movilidad espacial}

Se comprende las acciones respecto del territorio como estrategias, es decir, como producto de la voluntad de los sujetos, 
orientadas hacia determinados fines individuales o colectivos, lo que no quiere decir que estas no estén al mismo tiempo condicionadas en alguna medida por estructuras sociales. En este sentido, se sigue acá la propuesta de Löw (2013), de que la dimensión espacial de los procesos sociales incluye tanto estructuras espaciales como acción espacial, en tanto caras de la misma moneda. El espacio así, es conceptualizado como una estructura ordenadora de la acción humana, y al mismo tiempo como una forma de acción en sí misma. En este sentido se busca averiguar de qué modo los profesionales jóvenes originarios de la región, frente a lo que perciben como características estructurales de su territorio de origen, construyen el espacio social tanto en términos de percepciones como de estrategias, ahondando en el vínculo entre estas dos dimensiones.

Lo anterior resulta relevante ya que a nivel internacional existe evidencia de que la dimensión espacial y territorial constituye un aspecto importante de las trayectorias de sujetos de clases medias, ya sea durante su acceso a la educación superior (Almeida, 2012) como durante su trayectoria laboral (Miles, Savage y Bülhman, 2011). En el caso brasileño, Almeida (2012) da cuenta de que las probabilidades de ingresar a universidades de elite son desiguales según territorio de origen. En el caso inglés, al analizarse trayectorias de hombres de clase media, el territorio de origen genera un efecto similar, y se adiciona a ello que las experiencias de movilidad espacial constituyen hitos demarcadores de etapas en las trayectorias que permiten favorecer la movilidad social ascendente, 0 adaptarse a una nueva posición social más alta (Miles, Savage y Bülhman, 2011).
Ahora bien, en Chile son escasos los estudios que abordan el vínculo entre percepciones subjetivas sobre los territorios y la acción en él, es decir, el vínculo entre lo que Soja denomina segundo y tercer espacio. Destaca en este sentido el trabajo de Rodrigo y Atienza (2014) quienes analizan las percepciones que estudiantes universitarios de diversas regiones del país tienen respecto Antofagasta, encontrando que estas se diferencian de forma significativa según territorio. Por otro lado, existe también evidencia de que también las percepciones políticas tienen diferencias significativas según territorio, no solo a nivel regional sino a nivel intra-regional (Delamaza, Thayer y Gaete, 2015). No obstante, no se cuenta con antecedentes de estudios nacionales que vinculen la movilidad espacial por estudios universitarios y la localización de profesionales, con las percepciones subjetivas respecto de los territorios.

A continuación se presentan algunas investigaciones que aunque no hacen necesariamente el vínculo mencionado, aportan de todas maneras antecedentes que permiten contextualizar el fenómeno en análisis, en los tres momentos que aquí se abordan.

En Chile la oferta de educación superior se encuentra altamente concentrada en términos territoriales, tanto a nivel nacional como a nivel intra-regional (Donoso, Arias, Weason y Frites, 2012). Ello naturalmente tiene como consecuencia que para acceder a este nivel educativo, los estudiantes deben migrar 0 desplazarse diariamente. De hecho, de acuerdo a Rolando y Lara (2015) durante el año 2014, más de 150.000 jóvenes migraron interregionalmente por estudios superiores, de los cuales $71 \%$ lo hizo por estudios universitarios. 
Estas migraciones tuvieron como destino principalmente las tres regiones con más oferta superior del país, que coincidentemente son las tres regiones con presencia de ciudades que pueden ser consideradas metropolitanas: Santiago, Valparaíso y Concepción. Esta migración inter-regional se ubica en una tensión región/país, y afecta a las ciudades intermedias desde las que un número importante de estudiantes, principalmente de clases medias, emigra. Existe evidencia de que este no es solo un fenómeno nacional, sino que pareciera reproducirse en otras ciudades intermedias latinoamericanas, como en el caso de la ciudad de Villa Gesell, en Argentina, en la que los jóvenes que provienen de clases medias migran a ciudades metropolitanas y aquellos de clases bajas acceden solo a la oferta de educación superior local que tiende a ser reducida y en rubros o áreas temáticas de escasa rentabilidad, o simplemente no prosiguen estudios y se insertan en el mercado laboral local (Abrantes y Felice, 2015).

Ahora bien, la estrategia de movilidad espacial por motivos de estudios universitarios puede asumir también la forma de viajes diarios. En el caso de regiones no metropolitanas, ello es más común entre los estudiantes que acceden a la oferta universitaria que las ciudades intermedias pueden ofertar, lo cual ha sido favorecido en Chile especialmente durante los últimos años, luego de la aplicación de la política de gratuidad en la educación universitaria, aunque como indica Riquelme (2017) a partir del análisis del caso de la región de la Araucanía en Chile, las condiciones de acceso a la educación universitaria también se ven afectadas por barreras espaciales que tendrían efectos en el rendimiento académico y en las formas de experimentar la vida universitaria y la vida en la ciudad en general. Esto supone ciertos desafíos para las universidades. De hecho, analizando el caso específico de la ciudad de Valparaíso, que recibe a gran parte de quienes migran por motivos de estudios universitarios en Chile, Álvarez, Silva y Soto (2009) dan cuenta de desigualdades en el emplazamiento en el espacio urbano que afectan a estudiantes que provienen de otras regiones y de orígenes sociales bajos. En suma, la movilidad espacial por estudios universitarios parece estar asociada a tensiones entre región y país y entre ciudades intermedias e hinterland.

Las tensiones mencionadas se observan también en la inserción laboral de profesionales. Desde la lógica del capital humano calificado, se ha dado cuenta de que la distribución espacial de profesionales universitarios en Chile es marcadamente desigual entre regiones y concentrada particularmente en la región metropolitana (Mideplan y CED, 2004). Estas desigualdades no responden tanto a diferencias sectoriales en las economías territoriales, como a diferencias de carácter ocupacional según territorio, es decir a patrones diferenciados de división espacial del trabajo, de acuerdo a Lufin y Atienza (2010). Los autores encuentran en su análisis que en la región Metropolitana se encuentran sobrerrepresentadas ocupaciones relacionadas con la toma de decisiones y más intensivas en conocimiento, como es el caso de miembros del poder ejecutivo y judicial y directores de empresas, así como profesionales científicos e intelectuales en general. Ello en desmedro de las ciudades no 
metropolitanas del país, en las que aparecen sobrerrepresentadas las ocupaciones de técnicos y operarios.

En línea con lo anterior, desde una perspectiva más sociológica, Mac-Clure, Barozet y Maturana (2014), dan cuenta de que la configuración de clases según tipos de territorios varía de forma importante. Las clases medias profesionales tienden a localizarse en ciudades metropolitanas en primer lugar, y luego en ciudades intermedias, más que en territorios semirurales o rurales.

Lo anterior resulta relevante en sí mismo, y también porque la desigual distribución espacial de profesionales podría explicar la mayor parte de la desigualdad salarial entre comunas chilenas (Chacon y Paredes, 2015), como también en otros países de América Latina (Cueva y Alvarado, 2017). Ante esta constatación, los autores de ambos estudios concluyen en que se hace necesario el diseño e implementación de políticas que promuevan la redistribución espacial de profesionales universitarios. En Chile se han realizado algunas propuestas en esta dirección (Saravia, 2012, y Von Baer, Torralbo, Saravia y Toloza, 2012), que no han sido aplicadas hasta ahora.

En suma, este trabajo analiza tres etapas de las trayectorias de profesionales (educación secundaria, acceso a educación universitaria e inserción laboral) que de acuerdo a la literatura mencionada, parecen constituirse en hitos demarcadores de estas, y que las bifurcan. Se entiende aquí entonces, que las trayectorias de vida de los sujetos tienden a no ser lineales, sino a desarrollarse en etapas divididas por bifuraciones. En el caso chileno el concepto de trayectorias bifurcadas acuñado por Frédéric De Coninck y Francis Godard, ha sido aplicado por Pinto (2014, 2016), dando cuenta de que las trayectorias de vida se componen tanto de momentos tranquilos en los que no existen mayores cambios, y momentos decisivos en los que el destino social de los sujetos se ve comprometido, generándose así bifurcaciones que configuran la dinámica de estratificación social. Esto es corroborado también por los hallazgos de Miles, Savage y Bühlmann (2011), al encontrar que cierta cohorte de hombres profesionales británicos al referirse a sus propias trayectorias de vida hacen uso también de una narrativa en base a hitos demarcadores. En el caso chileno, estos puntos inflexión, se encuentran asociados a elementos propios del ámbito personal (el inicio de un matrimonio), educativo (el inicio de una carrera profesional), o laboral (un cambio de lugar de trabajo), entre otros (Guichard, Concha, Henríquez, Cavalli y Lalive d'Epinay, 2013). Se propone aquí, que la movilidad espacial es también un importante elemento en dichas bifurcaciones, en al menos tres etapas: 1) una primera que es posible asociar a la adolescencia y en la que se desarrollan los estudios secundarios o de enseñanza media de los sujetos. En esta etapa la bifurcación se produciría en el hecho de migrar o no por dichos estudios. Considerando la etapa del ciclo vital de los sujetos, la migración en este momento es principalmente una estrategia inter-generacional; 2) la segunda asociada a una etapa de juventud con mayor independencia respecto de la generación precedente, y que coincide con el acceso a la educación superior. En ella la bifurcación se produciría en la migración fuera de la región para acceder a la educación universitaria, que puede ser considerada una estrategia en la que los sujetos tienen mayor poder de decisión 
que en la anterior; 3) la tercera se asocia a la etapa adulta de los sujetos, una vez finalizados los estudios universitarios. La bifurcación aquí se produce en la decisión de localización como profesional. Quienes han cursado estudios universitarios en su región de origen pueden permanecer en ella o no, así como quienes han estudiado en otras regiones, pueden retornar o no. Ambas opciones tendrán efectos no solo materiales, sino también subjetivos. Así mismo, se propone que optar por una u otra acción espacial, está mediado por la subjetividad respecto de los territorios de origen.

\section{Supuestos}

Los supuestos que se buscan probar son en primer lugar, que las percepciones territoriales de los sujetos se refieren tanto a tensiones en distintos niveles: entre la región de Los Lagos y las regiones más centrales del país, como a tensiones entre las ciudades intermedias de la región de Los Lagos y sus correspondientes hinterland. Es decir, se plantea que las percepciones territoriales de los sujetos dan cuenta de la existencia de fuerzas espaciales que afectan su vida, en al menos estos dos niveles.

Como segundo supuesto a probar, se propone que los sujetos perciben estas fuerzas tanto en la etapa de su enseñanza media, acceso a la universidad, como en la etapa de inserción laboral. En tercer lugar se propone que las percepciones territoriales de los sujetos dan cuenta de fuerzas centrífugas que favorecen el despliegue de estrategias de movilidad espacial desde hinterland a ciudades intermedias, y desde la región de Los Lagos hacia regiones con presencia de ciudades metropolitanas.

Se propone como cuarto y último supuesto a corroborar que las percepciones territoriales subjetivas asociadas a las fuerzas que actúan en los distintos niveles y momentos mencionados, se refieren no sólo a aspectos de la dimensión física o tangible de los territorios, tales como infraestructura pública y privada, acceso a servicios, mercado laboral, facilidades para la movilidad física, entre otros. Sino que también a aspectos de naturaleza inmaterial de los territorios, como emociones vinculadas al espacio, elementos simbólicos, y aspectos culturales propios de ciertos grupos sociales con arraigo en territorios específicos.

Ahora bien, cuando se propone que las percepciones subjetivas sobre el territorio dan cuenta de fuerzas (centrífugas/centrípetas) que actúan en distintos niveles (región/país y ciudad/hinterland) y momentos (acceso a universidad/inserción laboral), ello no quiere decir que los sujetos necesariamente se refieran de forma explícita a dichas fuerzas o que sean conscientes de ellas, sino que en sus relatos dan indicios de la expresión de estas en los distintos niveles espaciales y en los distintos momentos aludidos. Es a través del análisis de los relatos de los entrevistados que se hace emerger los significados colectivos de las subjetividades individuales.

\section{Metodología}

Se utilizó una metodología cualitativa, con énfasis comprensivo, a partir de entrevistas 
semiestructuradas. Se entrevistó a 43 profesionales universitarios que rindieron la Prueba de Selección Universitaria (PSU) entre los años 2004 y 2006 en comunas de la región de Los Lagos y que residían también en dicha región en ese periodo de tiempo. La elección de este periodo se fundamenta en que de esta manera se logró identificar personas que en la actualidad tienen entre 4 y 8 años de experiencia laboral, tiempo que se estimó suficiente como para tener una aproximación adecuada a las tendencias en la localización espacial en la inserción laboral. Otros criterios para la incorporación a la muestra fue que hayan sido seleccionados en alguna carrera profesional de universidades adscritas al Sistema Único de Admisión (SUA), aunque no todos quienes fueron seleccionados en dichas universidades, finalizaron sus estudios allí, sino que algunos hicieron cambio a universidades privadas en el proceso de formación.
Además, en la selección de los entrevistados se consideraron dos grupos: por un lado se agruparon las ciudades intermedias, en contraste con el resto de comunas de la región, consideradas zonas bajo el influjo del primer grupo, como se fundamentó anteriormente. Para determinar el territorio de origen se consideró que los entrevistados hayan rendido la PSU en la comuna donde cursaron su enseñanza media.

El número de entrevistados en cada caso, se ajustó lo más posible a la proporción de seleccionados en universidades adscritas al SUA según tipo de territorio, en el periodo analizado. Se cumplió con este criterio con fines de coherencia en el muestreo, aunque sin pretensiones de representatividad, dado el enfoque cualitativo utilizado. A continuación se detallan las características de la muestra.

Las entrevistas fueron realizadas de forma presencial en la mayor parte de los

Cuadro 1 - Características de la muestra

\begin{tabular}{|c|c|c|c|c|}
\hline & & \multicolumn{2}{|c|}{ Lugar de origen en la región } & \multirow{2}{*}{ Total } \\
\hline & & Ciudad intermedia & Hinterland & \\
\hline \multirow{2}{*}{$\begin{array}{c}\text { Lugar de estudios } \\
\text { universitarios }\end{array}$} & En la región & 6 & 3 & 9 \\
\hline & Fuera de la región & 26 & 8 & 34 \\
\hline \multirow{2}{*}{ Residencia actual } & En la región & 13 & 5 & 18 \\
\hline & Fuera de la región & 19 & 6 & 25 \\
\hline \multicolumn{2}{|c|}{ Total } & 32 & 11 & 43 \\
\hline
\end{tabular}

Fuente: elaboración propia. 
casos. El resto fueron hechas utilizando el software de comunicación online Skype o el servicio de Google, Hangout - en el caso de entrevistados residentes fuera de Chile y regiones lejanas. Las entrevistas tuvieron una duración que fluctuó entre 60 y 90 minutos aproximadamente. Fueron grabadas en audio, posteriormente transcritas y analizadas con ayuda del software Atlas.ti. Los códigos fueron creados a medida que se leían las entrevistas utilizando la técnica de comparación constante (Gibbs, 2012). Una vez codificada la unidad hermenéutica se agruparon los códigos en familias. Posteriormente, el análisis se llevó a cabo a partir de dos lógicas, una centrada en lo numérico y otra más comprensiva. La primera implicó contar las codificaciones de cada familia en las dos muestras y calcular proporciones, con el fin de identificar tendencias en los relatos de ambos grupos. La segunda consistió en una lectura atenta de las citas de cada una de las familias para identificar los principales argumentos comunes y diferenciaciones entre muestras. Este procedimiento puede ser considerado como triangulación de métodos (Gibbs, 2012). Para efectos de la presentación de los resultados, se indican las principales tendencias desde una lógica comprensiva.

\section{Resultados}

Una primera observación general es que las dos tensiones en análisis se diferencian según el momento vital de los sujetos entrevistos. La tensión ciudad intermedia/hinterland es relevada cuando los entrevistados hacen referencia a la etapa de la enseñanza media, mientras que la tensión región de Los Lagos/ regiones metropolitanas se asocia a las etapas de acceso a la universidad y de inserción laboral.

El análisis a continuación separa los distintos momentos, distinguiendo qué tensiones espaciales preponderan en estos, e identificando al interior de cada uno de ellos los principales elementos que pueden ser considerados indicadores de fuerzas centrifugas y centrípetas en las percepciones subjetivas sobre los territorios, y su asociación con estrategias concretas de movilidad espacial llevadas a cabo por los sujetos.

\section{Etapa de educación media}

En la etapa de educación media prepondera la tensión entre ciudades intermedias e hinterland. Los discursos de los entrevistados enfatizan dos elementos que pueden ser considerados como elementos propios de fuerzas centrífugas en desmedro de las comunas bajo la esfera de influencias de ciudades intermedias. Uno de ellos es la percepción de que la calidad de la educación impartida en estas ciudades es mejor que la de sus hinterland. El segundo hace referencia a que los contactos sociales tendrían un capital cultural más amplio en las ciudades, y más limitado en los hinterland.

En el primer elemento la percepción subjetiva involucra no solo a los sujetos entrevistados, sino que a sus padres, que favorecen el despliegue de estrategias de movilidad inter-comunal por motivos de estudios secundarios: 
El liceo de Entre Lagos no tenía muy buena reputación y afortunadamente mis papás podían costearme un colegio en Osorno, así que estudie la media allá. (Hombre, originario de Entre Lagos, estudió universidad en Santiago, reside en Santiago)

En efecto, estas estrategias son comunes en la región de Los Lagos (Donoso y Arias, 2012). Tienen un carácter inter-generacional y se orientan a la búsqueda de movilidad social.

El segundo elemento consiste en un juicio valorativo respecto del capital cultural de las relaciones que se dan entre pares en las zonas semirurales. Este es considerado limitado, y constituye una razón por la que preferir estudiar en una ciudad intermedia. El relato a continuación es hecho por una mujer que estudió parte de su enseñanza media en Osorno, pero tuvo que finalizarla en su comuna de origen, haciéndosele patente el contraste cultural entre sus compañeros en uno y otro territorio:

[...] el perfil de estudiante que llegaba al Liceo eran la gran mayoría gente de campo [...] y yo estaba acostumbrada a otro mundo, con otras conversaciones sobre arreglar el mundo, el cambio climático... En cambio al llegar allá, mis compañeros me hablaban de la lechadura de las vacas, de sembrar papas [...] Entonces yo encontraba tan limitado lo que podía conversar con ellos. (Mujer, originaria de Entre Lagos, estudió universidad en Los Lagos, reside en Osorno)

En suma, en lo referido a la tensión ciudad intermedia/hinterland, se encuentra que esta es relevada en la etapa de enseñanza secundaria por parte de quienes son originarios del segundo tipo de territorios, contiene solo elementos propios de fuerzas centrifugas que van en desmedro de los hinterland, y no responden a aspectos económicos, sino a elementos culturales y de oferta educativa. Además, se observa que las percepciones se asocian de forma concreta a estrategias de movilidad inter-comunal por motivos de estudios secundarios desde hinterland a ciudades intermedias que los entrevistados indican haber llevado a cabo.

\section{Etapa de formación universitaria}

En la etapa de formación universitaria prepondera la tensión entre la región de Los Lagos y regiones metropolitanas. Concretamente se hace referencia a la diversidad cultural, y la oferta recreativa.

Se releva la diversidad cultural como un elemento importante en la valoración territorial, favoreciéndose a las regiones metropolitanas por sobre la de origen. Es decir, estas últimas tendrían una mayor diversidad cultural y tolerancia a la diversidad, lo que es positivamente valorado por los entrevistados. Un entrevistado originario de Osorno que cursó su educación universitaria en Concepción indica:

(en la región de Los Lagos) todo el mundo es igual, todo el mundo se viste igual, como que andan en la misma onda. Si uno llama la atención de alguna forma eres apuntado con el dedo [...] En Concepción no pasa eso, hay gente haciendo cuestiones, llamando la atención. (Hombre, originario de Osorno, estudió universidad en Concepción, reside en Osorno) 
Se asocia esta más limitada diversidad a que las ciudades de la región de Los Lagos, a pesar de contar con grupos de población de origen inmigrante europeo, no logran el cosmopolitismo que Santiago. De hecho, los grupos de origen europeos no favorecerían la diversidad, ya que son asociados a la vida rural, y mencionados como tradicionalistas:

Santiago es más cosmopolita. Llega gente de todas partes, más instruida, tienes más estudios, más cultura, más conocimientos. Acá todavía hay gente muy ignorante, aunque suene feo, pero si, hay mucha gente como a la antigua. Yo creo que es porque se criaron en el campo, o (debido a que) las familias alemanas tienen otras tradiciones. (Mujer, originaria de Puerto Montt, estudió universidad en Santiago, reside en Puerto Montt)

También en la etapa de estudios universitarios, se releva la desigual oferta recreativa entre la región de origen y las regiones metropolitanas. Estas últimas serían mucho más atractivas para los entrevistados, especialmente en su etapa de juventud, lo que queda reflejado en el siguiente relato de un alguien originario de Osorno que cursó su educación universitaria en Concepción:

Si no estabas con tus amigos y no inventabas algo, no había ningún lugar donde ir, hubo una época en donde no había cine, eran detalles que hacían de Osorno una ciudad que, no me desagradaba porque el estilo de vida también es muy cómodo en Osorno, pero buscaba un poco más de diversión. Cuando uno es chico quiere salir y divertirse. (Hombre, originario de Osorno, estudió universidad en Concepción, reside en Osorno)

\section{Etapa de inserción laboral}

La etapa de inserción laboral también se asocia a la tensión entre región de Los Lagos y regiones metropolitanas. Los relatos dan cuenta predominantemente de fuerzas centrípetas que actúan en desmedro de la región de origen. Se hace referencia a la oferta laboral del territorio, a la calidad de las relaciones sociales y proximidad con familia extendida, contacto con la naturaleza y la calidad de vida urbana en general.

En primer lugar, se plantea que la región de origen no tienen una oferta de empleo suficientemente amplia y diversa como para que quienes desean retornar a ella, puedan trabajar allí. Resulta interesante en este sentido, que la tendencia general es a desear retornar luego de haber cursado estudios universitarios en regiones metropolitanas, pero esto se ve truncado porque la estructura ocupacional no ofrece suficientes posibilidades reales de insertarse en el territorio de origen, lo que queda reflejado en el siguiente relato:

El tema es cuando tú quieres volver y no está la oportunidad, y tienes que irte. Da pena. He visto amigos, primos y familiares que han llegado con su cartón (certificado universitario) acá, con muchas expectativas, y no ha resultado... Y tienen que obviamente irse al continente, pero siempre con un ojo allá y el otro acá, porque uno igual tiene las ganas de quedarse para siempre acá. (Hombre, originario de Castro, estudió universidad en Temuco, reside en Castro)

Ahora bien, aunque no insertarse laboralmente en el territorio de origen se asocia principalmente a la razón expuesta, 
algunos entrevistados indican que el volver es postergado para ganar experiencia en ambientes de mayor competitividad en regiones metropolitanas, $u$ otros indican que su formación al ser demasiado especializada, sería subutilizada en sus territorios de origen, dada la estructura ocupacional de estos. De esta manera, las diferencias en cantidad y calidad de la oferta laboral de la región de origen forma parte de fuerzas centrifugas en desmedro de esta, y se asocian a estrategias concretas de movilidad espacial inter-regional que los entrevistados indican haber llevado a cabo.

Los entrevistados refieren también tres elementos que pueden ser considerados los principales indicadores de fuerzas centrípetas asociadas a la inserción laboral. Se enfatiza que en la región de origen las relaciones sociales tienen una mayor calidad, tanto las familiares, como las relaciones en general. Dicha calidad se asocia a una mayor densidad en las interacciones, y a una mayor permanencia en el tiempo:

(en Santiago las relaciones) son menos trascendentales, son menos longevas en el tiempo diría yo, las amistades que al menos conmigo han perseverado en el tiempo son aquellas que yo he formado con gente de acá. (Hombre, originario de Osorno, estudió universidad en Santiago, reside en Osorno)

Yo creo que lo que prima en el sur son los valores de la familia, y no la familia nuclear sino en el amplio sentido, quien haces tú tu familia, tus amigos, la patota, aquí como que se vive todo en gran comunidad: los horarios de comida, las juntas... (Mujer, originaria de Ancud, estudió universidad en Temuco, reside en Ancud)
Se enfatiza también que en la región de Los Lagos se tiene un contacto más estrecho con la naturaleza que en las ciudades metropolitanas. Esto es valorado positivamente:

A mí me gusta vivir acá. En realidad cuando he ido a Santiago extraño la tierra, el olor de la tierra mojada, lo verde. Me gusta vivir acá. (Mujer, originaria de Osorno, estudió universidad en Osorno, reside en Osorno)

Te da un cierto estatus vivir en Puerto Varas que quizás no está reflejado en ganar un buen sueldo, pero si tener una buena calidad de vida. Por ejemplo, de mi casa yo camino tres cuadras y llego al lago, y puedo caminar tranquilamente y llego al centro, y de mi casa al centro de la ciudad son quince minutos. (Hombre, originario de Puerto Varas, estudió universidad en Osorno, reside en Puerto Varas)

Por último, asociado a lo anterior, se menciona que la región de origen permite tener una vida más tranquila que en las ciudades metropolitanas, lo que también es valorado positivamente:

yo nací en Santiago (y viví ahí) hasta los
doce años, y luego nos vinimos a Osorno.
Me gustó el cambio, el poder caminar
tranquilo, las facilidades que tiene una
ciudad chica en que podemos ir de la casa
al centro caminando. (Hombre, originario
de Osorno, estudió universidad en Osorno,
reside en Osorno)

Esta tranquilidad mencionada no solo se debe a las distancias caminables en una ciudad intermedia, sino que también es asociada por los entrevistados a menores niveles de delincuencia en contraste con ciudades más grandes. 


\section{Cuadro 2 - Tensiones entre fuerzas centrífugas y centrípetas en distintos niveles territoriales y momentos}

\begin{tabular}{|l|l|l|}
\cline { 2 - 3 } \multicolumn{1}{c|}{} & \multicolumn{1}{c|}{ Tensión } & \multicolumn{1}{c|}{ Elementos que componen la tensión } \\
\hline Educación media & $\begin{array}{l}\text { Ciudad intermedia versus } \\
\text { hinterland }\end{array}$ & $\begin{array}{l}\text { Fuerzas centrifugas: } \\
\text { - Diferencias inter-comunales en calidad educacional } \\
\text { - Diferencia en bagaje cultural en relación entre pares }\end{array}$ \\
\hline Educación universitaria & $\begin{array}{l}\text { Región de origen versus } \\
\text { regiones metropolitanas }\end{array}$ & $\begin{array}{l}\text { Fuerzas centrífugas: } \\
\text { - Diferencias en grado de diversidad cultural y tolerancia a la diversidad } \\
\text { - Diferencias en oferta recreativa }\end{array}$ \\
\hline Inserción laboral & $\begin{array}{l}\text { Fuerzas centrífugas: } \\
\text { Región de origen versus }\end{array}$ & $\begin{array}{l}\text { - Diferencias en oferta laboral } \\
\text { regiones metropolitanas centrípetas: } \\
\text { - Calidad de las relaciones sociales y proximidad con familia extendida } \\
\text { - Contacto con la naturaleza } \\
\text { - Calidad de vida urbana }\end{array}$ \\
\hline
\end{tabular}

Fuente: elaboración propia.

A continuación, la Tabla 2 resume el conjunto de tensiones entre fuerzas centrífugas y centrípetas en cada uno de los momentos y las tensiones consideradas.

\section{Reflexiones finales}

El análisis desarrollado permite corroborar los supuestos planteados: las percepciones territoriales se asocian a estrategias concretas de movilidad espacial, de acuerdo al relato de los entrevistados. En efecto, en los tres momentos analizados, los entrevistados indicaron haber desplegado estrategias de movilidad espacial que concordaban con las principales tendencias en cuanto a percepciones subjetivas del territorio. Estas percepciones y estrategias son productos de tensiones que tienen lugar en distintos niveles territoriales, y varían según momentos de la vida de los sujetos. A medida que se desarrolla el ciclo vital de los sujetos, las tensiones tienen que ver con escalas espaciales mayores. Algunos de los entrevistados hacen referencia inclusive a un nivel espacial internacional, fundamentalmente asociado a estudios de postgrado, que resultaría interesante de analizar en futuras investigaciones. Por último, se corrobora que los contenidos de las percepciones territoriales que componen las tensiones entre fuerzas centrifugas y centrípetas, no solo hacen referencia a aspectos económicos o laborales, sino que predominantemente se refieren a aspectos de orden cultural, social y propiamente de vínculo con el espacio urbano.

A la luz del análisis desarrollado, parece relevante incorporar al análisis desde el enfoque de la trialectica del espacio planteado por Soja, una perspectiva temporal o de trayectoria en la vida de los sujetos, ya que en 
efecto, al menos en lo observado en la muestra utilizada, las diversas desigualdades y tensiones territoriales van variando en cuanto al nivel en que estas operan, en cuanto a los contenidos de las percepciones territoriales, y en cuanto a las estrategias posibles de desplegar. En este sentido, a medida que avanza la trayectoria de los sujetos, las percepciones y acciones respecto del territorio se van superponiendo una sobre otra, generando recorridos diversos de percepciones/acciones en cuanto a los territorios. Es decir, la bifurcación producida en la etapa de enseñanza media condiciona las posibilidades de acción en la bifurcación de la educación universitaria, y a su vez, la bifurcación producida en esta condiciona las posibilidades en la etapa de inserción laboral. Por ejemplo, como mencionaba la narración de uno de los entrevistados, el hecho de desplazarse diariamente entre comunas para acceder a lo que se considera como una mejor educación durante la etapa de educación media, es visto como crucial para obtener un mejor puntaje PSU que, a su vez, permitirá tener un margen más amplio de elección de carreras, universidades, becas y mayores posibilidades de migrar inter-regionalmente en la etapa de formación universitaria. Adicionalmente, otros entrevistados indican que la posibilidad de insertarse laboralmente en ciertos puestos laborales en regiones metropolitanas está condicionada no solo por la carrera estudiada, sino por la universidad en la que se estudió, reconociéndose que las universidades de elite que concentran mayor prestigio (capital simbólico y social en la lógica de Bourdieu) se ubican en la región metropolitana. Se trata de las estrategias de clausura de clase con las que se ven enfrentados quienes han ascendido a la clase de servicio alta, pero que no nacieron en ella (PNUD, 2017). De esta manera, las trayectorias de los profesionales se configuran a partir de una serie de causas y efectos en bifurcaciones en las que la dimensión espacial resulta crucial. Una futura investigación que siga esta lógica debiera clasificar tipos reconocibles de trayectorias. Ello debiera necesariamente contemplar que las trayectorias variarán según cohortes, dada la evolución en el tiempo de las condiciones materiales de los territorios.

En consonancia con lo anterior, resulta relevante destacar que como se desprende del análisis realizado, el rol de la ciudad intermedia resulta variable y varía de acuerdo al momento de la vida de los entrevistados. Resulta crucial para quienes desde sus hinterland migran inter-comunalmente por estudios secundarios con el fin de obtener lo que consideran una mejor educación, y acceder a un mayor capital cultural y social, pero en las etapas de acceso a la universidad y posterior inserción laboral, el valor de las ciudades intermedias se ve mermado, ya que no logran satisfacer los requerimientos diversos de los sujetos en trayectorias de profesionalización, quienes optan predominantemente por migrar a ciudades metropolitanas. Esto puede ser explicado principalmente por la estructura ocupacional de las ciudades chilenas, aunque debe considerarse que esta ha mutado de forma considerable en los últimos veinte años, a partir de la aplicación del modelo neoliberal en el país, haciéndose cada vez más homogéneas las ciudades intermedias mediante su "tercerización", es decir, mediante el incremento relativo de la importancia de las ocupaciones asociadas a servicios y la 
consecuente disminución de las actividades laborales de carácter primario (Fuentes, Link y Valenzuela, 2017). Ese es el caso de Osorno, que pareciera estar en un proceso de acrecentamiento de oportunidades laborales para profesionales en el área de servicios, no necesariamente asociados a actividades primarias. En este sentido, Fuentes, Link y Valenzuela (2017) dan cuenta de una cierta tendencia a la urbanización de las ciudades intermedias en Chile, lo que por un lado podría facilitar la inserción de profesionales en estas ciudades, pero por otro lado, genera una serie de interrogantes: ¿Es la metropolización de las ciudades intermedias la solución para la fuga de profesionales originarios de estas, 0 es posible pensar formas de fortalecimiento de estas ciudades sin la pérdida de lo que los entrevistados consideran sus elementos más valorables (buenas relaciones sociales, proximidad con la naturaleza, buena calidad de vida en general)?

[I] https://orcid.org/0000-0003-3196-7831

Universidad del Bio Bio, Departamento de Ciencias Sociales. Concepción, Chile.

felipe.saravia.cortes@gmail.com

\section{Referencias}

ALMEIDA, M. (2012). "As desigualdades históricas e o início do processo de expansão e democratização do acesso ao sistema de Ensino Superior no Brasil". In: BRONZO, C. et al. (org.). Desigualdades Educacionais e Pobreza. Belo Horizonte, Ed. Pucminas.

ÁLVAREZ, L.; SILVA, L. y SOTO, M. (2009). Dimensión espacial de la movilidad cotidiana universitaria: el caso del gran Valparaíso. Revista INVI, v. 24, n. 65.

BELLET, C. y LLOP, J. M. (2004). Miradas a otros espacios urbanos: las ciudades intermedias. Scripta Nova, v. 8, n. 165.

BERDEGUÉ, J.; JARA, B.; FUENTEALBA, R.; TOHÁ, J.; MODREGO, F.; SCHEJTMAN, A. y BRO, N. (2011). Territorios funcionales en Chile. Centro Latinoamericano para el Desarrollo Rural (RIMISP), Documento de Trabajo, n. 102. Santiago, Chile.

BOURDIEU, P. (2006). La identidad y la representación: elementos para una reflexión crítica sobre la idea de región. Ecuador Debate, n. 67, pp. 165-184.

CASADO-DÍAZ, J. y COOMBES, M. (2011). The delineation of 21st century local labour market areas: a critical review and a research agenda. Boletín de la Asociación de Geógrafos Españoles, n. 57, pp. 7-32. 
CASADO-DÍAZ, J., MARTÍNEZ-BARNEBÉU, L. y ROWE, F. (2017). An evolutionary approach to the delimitation of labor market areas: an empirical application for Chile. Spatial economic analysis, v. 12.

CHACÓN, S. y PAREDES, D. (2015). Desigualdad espacial de ingresos en Chile y su relación con la concentración de capital humano. El trimestre económico, v. 82, n. 326, pp. 351-377.

COOMBES, M. y BOND, S. (2008). Travel-to-Work Areas: the 2007 review. Londres, Office for National Statistics. Disponible en: http://www.istat.it/it/files/2014/12/final_TTWA_report.doc. Acceso en: 22 sep 2017.

CUEVA, K. y ALVARADO, R. (2017). Concentración espacial de capital humano calificado y desigualdad regional de ingresos en Ecuador durante el periodo 2003-2012. Paradigma Económico, v. 9, n. 1, pp. 5-26.

DE ABRANTES, L. y FELICE, M. (2015). ¿Ciudad sin jovenes o jovenes sin ciudad: reflexiones sobre el derecho a la ciudad en jovenes que habitan en ciudades intermedias. Cuaderno Urbano, v. 19, n. 19, pp. 115-136.

DELAMAZA, G., THAYER, L. y GAETE, J. M. (2015). Diferencias territoriales en las percepciones políticas en Chile: una aproximación operacional a la escala nacional y regional. Si Somos Americanos, v. 15, n. 1, pp. 15-48.

DONOSO, S.; ARIAS, O.; WEASON, M. y FRITES, C. (2012). La oferta de educación superior de pregrado en Chile desde la perspectiva territorial: inequidades y asimetrías en el mercado. Calidad en la educación, n. 37, pp. 99-127.

DONOSO, S. y ARIAS, O. (2012). Distribución desigual de las oportunidades educativas en el territorio y migración de la matrícula escolar: el caso de la región de Los Lagos (Chile). Estudios pedagógicos, v. 38, n. 2 , pp. 35-54.

FUENTES, L.; LINK, F. y VALENZUELA, F. (2017). Impactos de la dinámica urbana en los mercados laborales en las principales ciudades chilenas. Cadernos Metrópole. São Paulo, v. 19, n. 38, pp. 157-177.

FUENTES, L.; MAC-CLURE, O.; MOYA, C. y OLIVOS, C. (2017). Santiago de Chile: ¿Ciudad de ciudades? Desigualdades sociales en zonas de mercado laboral local. Cepal Review, n. 121, pp. 93-109.

GIBBS, G. (2012). El análisis de datos cualitativos en investigación cualitativa. Madrid, Ediciones Morata.

GUICHARD, E.; CONCHA, V.; HENRÍQUEZ, G.; CAVALLI, S. y LALIVE D’EPINAY, C. (2013). Reconstrucción subjetiva del curso de la vida en Chile. Revista Mexicana de Sociología, v. 75, n. 4, pp. 617-646.

HIERNAUX, D. (2007). Los imaginarios urbanos: de la teoría y los aterrizajes en los estudios urbanos. Eure, v. 33, n. 99, pp. 17-30.

INSTITUTO NACIONAL DE ESTADÍSTICAS DE CHILE [INE] (2017). Proyección de población por comuna a partir de censo de 2002. Gobierno de Chile, Santiago, Chile.

JORDÁN, R. y SIMIONI, D. (1998). Ciudades intermedias en América Latina y el Caribe: propuestas para la gestión urbana. Comisión Económica Para América Latina y el Caribe (Cepal). Santiago, Chile.

LÖW, M. (2013). O spatial turn: para uma sociologia do espaço. Tempo Social, v. 25, n. 2, pp. 17-34. 
LUFín, M. y ATIENZA, M. (2010). Diferencias entre la composición sectorial y ocupacional de las principales ciudades chilenas. EURE, v. 36, n. 108, pp. 75-93.

(2015). "El papel de las ciudades intermedias en la red de flujos de conmutación en Chile". In: MATURANA, F. y ROJAS, A. (eds.). Ciudades intermedias en Chile. Territorios olvidados. Santiago/ Chile, RIL Editores.

MAC-CLURE, O. y CALVO, R. (2013). Desigualdades sociales y tipos de territorios en Chile. Polis, n. 34, pp. $467-490$

MAC-CLURE, O.; BAROZET, E. y MATURANA, V. (2014). Desigualdad, clase media y territorio en Chile: ¿clase media global o múltiples mesocracias según territorios? EURE, v. 40, n. 121, pp. 163-183.

MATURANA, F. (2015). “¿Ciudad media o ciudad intermedia? Evolución conceptual y estudio en Chile". In: MATURANA, F. y ROJAS, A. (eds.). Ciudades intermedias en Chile. Territorios olvidados. Santiago de Chile, RIL Editores.

(2016). Ciudades intermedias en Chile: definición de un territorio en transición. Planeo, n. 27.

MATURANA, F. y ARENAS, F. (2012). El policentrismo en Chile: medición exploratoria para el sistema de ciudades de las regiones de La Araucanía, de Los Ríos y de Los Lagos. Norte Grande, n. 52, pp. 37-56.

MILES, A.; SAVAGE, M. y BÜHLMANN, F. (2011). Telling a modest story: accounts of men's upward mobility from the National Child Development Study. The British Journal of Sociology, n. 62, pp. 418-441.

MINISTERIO DE DESARROLLO Y PLANIFICACIÓN NACIONAL (MIDEPLAN); CENTRO DE ESTUDIOS DEL DESARROLLO (CED) (2004). Distribución del capital humano en Chile. Santiago, Chile.

MORALES, F. (2012). La geografía de la percepción: una metodología válida aplicada al caso de una ciudad tipo medio-pequeño. El ejemplo de Yecla (Murcia). Papeles de Geografía, n. 55-56, pp. 137-152.

ORGANIZACIÓN DE LAS NACIONES UNIDAS PARA LA EDUCACIÓN - UNESCO (1999). Ciudades intermedias y urbanización mundial. Ajuntament de Lleida. Unesco, Lleida.

PINTO, C. (2014). Estudiar un posgrado en el extranjero ¿una migración previsible? Sociedad \& Equidad, n. 6, pp. 214-236.

(2016). Reflexiones sobre la inserción laboral de doctores en universidades chilenas. Fronteras, v. 3, n. 1, pp. 109-124.

PROGRAMA DE LAS NACIONES UNIDAS PARA EL DESARROLLO - PNUD (2017). Desiguales: orígenes, cambios y desafíos de la brecha social en Chile. Santiago de Chile, Uqbar editores.

RIQUELME, H. (2017). Cruzando La Araucanía (Chile). Un análisis de las prácticas de movilidad cotidiana de universitarios adscritos a la gratuidad. Cultura-hombre-sociedad, v. 27, n. 1, pp. 23-50.

RODRIGUEZ, J.; GONZÁLEZ, D. y OJEDA, M. (2009). “Evolución del sistema de ciudades de Chile durante la segunda mitad del siglo XX y de sus flujos de migración en los tres últimos censos: ¿continuidad o cambio?" In: HIDALGO, R.; MATTOS, C. y DE ARENAS, F. (eds.). Chile: del país urbano al país metropolitano. Santiago de Chile, Pontificia Universidad Católica de Chile.

RODRIGO, L. y ATIENZA, M. (2014). Migración y representaciones regionales: discursos sobre la Región de Antofagasta. Eure, v. 40, n. 120, pp. 159-181. 
ROLANDO, R. y LARA, A. (2015). Movilidad de Educación Superior desde Región de Origen. Reporte del Sistema de Información de Educación Superior - SIES. Chile, Ministerio de Educación.

ROWE, F.; CASADO-DÍAZ, J. y MARTÍNEZ-BARNEBÉU, L. (2017). Functional labor áreas for Chile. Region, v. 4, n. 3, pp. 7-9.

SARAVIA, F. (2012). ¿Cómo aumentar stocks de capital humano calificado a nivel local?: políticas públicas comparadas. Revista Desenvolvimento Regional em Debate, v. 2, n. 2, pp. 63-89.

SOJA, E. (2008). Postmetrópolis: Estudios críticos sobre las ciudades y las regiones. Madrid, Traficantes de Sueños.

TAULELLE, F. (2015). “A la búsqueda de las ciudades intermedias. Algunos elementos de discusión”. In: MATURANA, F. y ROJAS, A. (eds.). Ciudades intermedias en Chile. Territorios olvidados. Santiago de Chile, RIL Editores.

VALICELLI, L. y PESCI, R. (2002). Las nuevas funciones urbanas: gestión para la ciudad sostenible. Santiago de Chile, Cepal.

VARA, J. L. (2008). Cinco décadas de Geografía de la percepción. Ería, n. 77, pp. 371-384.

VON BAER, H.; TORRALBO, F.; SARAVIA, F. y TOLOZA, I. (2012). Propuesta de un sistema regional integrado de gestión de capital humano calificado para el desarrollo de las comunas y regiones de Chile. In: III ENCUENTRO DE LA SOCIEDAD CHILENA DE POLÍTICAS PÚBLICAS. Santiago de Chile, Universidad de Chile.

WACQUANT, L. (2017). Bourdieu viene a la ciudad: Pertinencia, principios, aplicaciones. Eure, v. 43, n. 129 , pp. 279-304.

Texto recebido em 22/set/2017

Texto aprovado em 10/abr/2018 
